\title{
The Potentialities of Electroless Gold Plating of Transistor Headers
}

\author{
Reginald K. Asher \\ Motorola Semiconductor Products Inc., Phoenix, AZ., U.S.A.
}

Less than a decade ago the semiconductor industry was electrodepositing $3 \mu \mathrm{m}$ of gold on the headers of small transistors such as the TO-18. This thickness of gold was necessary for bonding of the die or semiconductor chip. Since then the price of gold has increased considerably and there has been great interest in the industry in techniques which would result in decreased gold use.

Savings have been realized in various ways. Thus, improvements in the technique of die-bonding achieved by increasing the thickness of gold on the die and by increasing the scrubbing action and speed of die-bonding machines have permitted a reduction in the thickness of the coating on headers without loss of product reliability. Epoxy die-bonding has also helped and gold thicknesses on headers can now be reduced to $0.25 \mu \mathrm{m}$ successfully. Selective brush plating of headers is also extensively applied (1). Against this background, it seems appropriate to place on record an approach to the problem which has been explored at Motorola, albeit with negative results.

One drawback in the electroplating of headers is the excessive deposition of gold on the high plating current density areas, such as the edges of eyelets and the ends of leads. It seemed possible that this might be avoided by using electroless methods for depositing the gold and that such methods might be suitable for applying coatings of uniform thicknesses up to $5 \mu \mathrm{m}$, which would be suitable not only for bonding of dies and thermocompression or ultrasonic bonding of wires, but also for the making of soldered bonds to military specifications (2).

Several nillion transistor headers, of which some typical examples are shown here, are produced throughout the world every dny. Such devices are plated witl gold to ensure ease of entectic bonding of the semiconductor chip to the top surface of the header. The intricate shape of these headers results in uneven electroplating current density and consequently in uneven gold distribution on them. Electroless gold plating which results mornally in coatings of uniform thickness was, therefore, a tempting alternative to electroplating

\section{Electroless Gold Plating}

Electroless methods for the deposition of gold fall into three categories: First, there are simple immersion techniques in which the substrate is dipped into a gold complex solution, from which the metal is plated out by a replacement reaction. The coatings are thin, since deposition ceases as soon as there is no more base metal exposed to the solution. Secondly, there is a wide range of procedures in which reducing reagents plus various additives are incorporated in the gold plating solution, and in which gold deposition by replacement is supplemented by gold deposition as a result of reduction. Thirdly, there are solutions from which gold is deposited autocatalytically (3).

An autocatalytic system suitable for the plating of transistor headers is not available on the market and attempts by the author to develop such a system have been unsuccessful. The potentialities of solutions of the first and second types were therefore explored for the purpose and coatings from them were compared with those from a commercial electrolytic bath.

\section{Experimental Procedures and Data}

A $50 \times 100 \mathrm{~mm}$ horizontal plating barrel with $4 \mathrm{~mm}$ diameter through holes was used for the 'electroless' and immersion solutions. A lot size of 200 TO-18 devices was used. The solution volume was 21 .

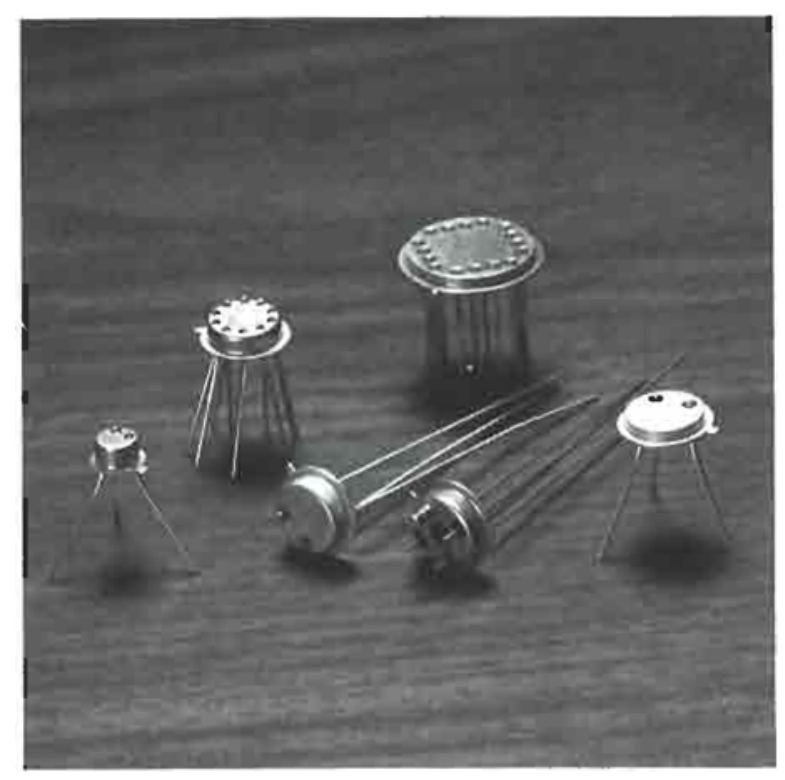



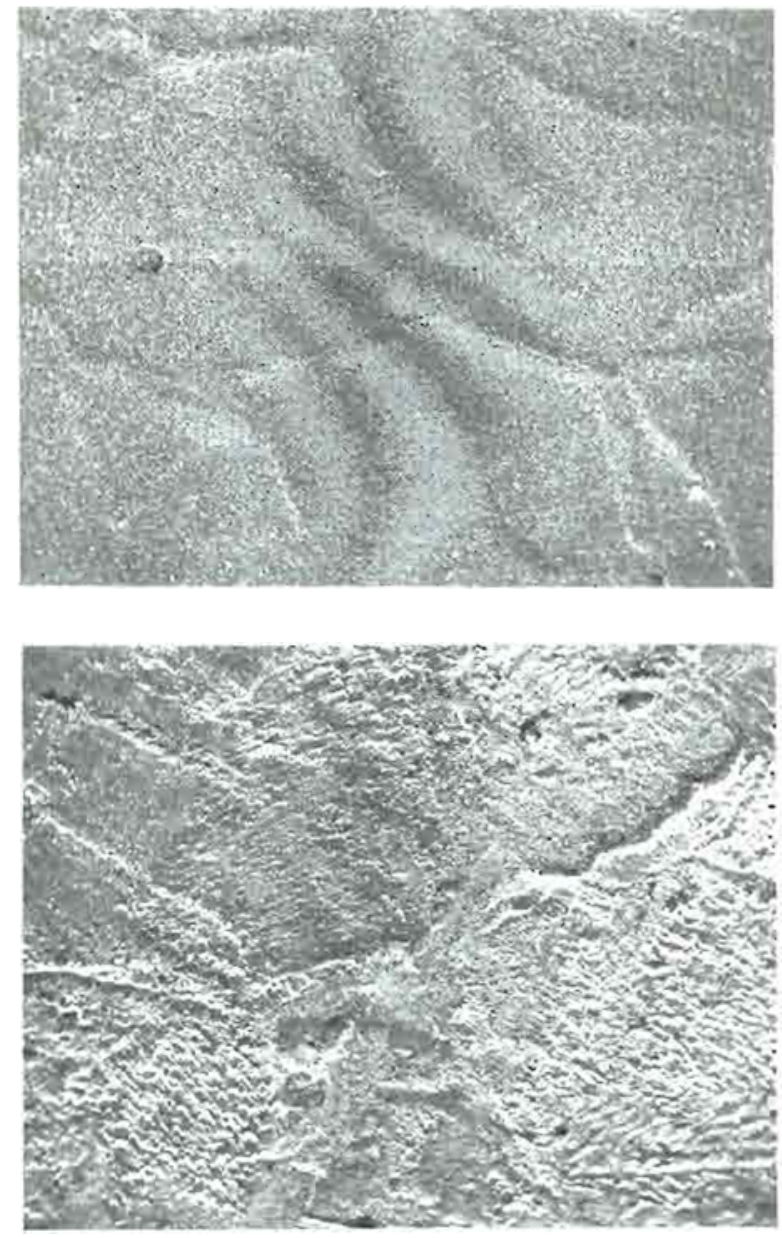

A standard production barrel and plating lot of 20000 TO- 18 headers was used with the electrolytic solution $(380 \mathrm{l})$. The barrel had a solid bar and a 150x600 mm cylinder.

The plating solutions used included:

(1) A proprietary electroless gold plating solution characterized by a gold content (as $\mathrm{KAu}(\mathrm{CN})_{2}$ ) of 2 to $4 \mathrm{~g} / \mathrm{l}$, a $\mathrm{pH}$ of 5.0 to 6.2 and a working temperature of 60 to $85^{\circ} \mathrm{C}$

(2) Motorola electroless gold plating solution $M$ characterized by a gold content of 6 to $10 \mathrm{~g} / \mathrm{l}$, a $\mathrm{pH}$ of 5.2 to 5.5 and a working temperature of 90 to $96^{\circ} \mathrm{C}$

(3) A proprietary immersion gold plating solution characterized by a gold content of $4 \mathrm{~g} / \mathrm{l}$, a $\mathrm{pH}$ of 3.5 to 4.0 and a working temperature of 60 to $65^{\circ} \mathrm{C}$

(4) A proprietary electrolytic gold plating solution characterized by a gold content of 7 to $9 \mathrm{~g} / \mathrm{l}$, a $\mathrm{pH}$ of 6.0 to 8.0 , a working temperature of 40 to $60^{\circ} \mathrm{C}$ and a density of 15 to $22^{\circ} \mathrm{Be}$.

The substrate surface in each instance was either Kovar or electroless deposited nickel-phosphorus.

The average thickness of deposits was determined by a weigh-strip-weigh procedure applied to 20 units. Local thickness measurements were made by beta

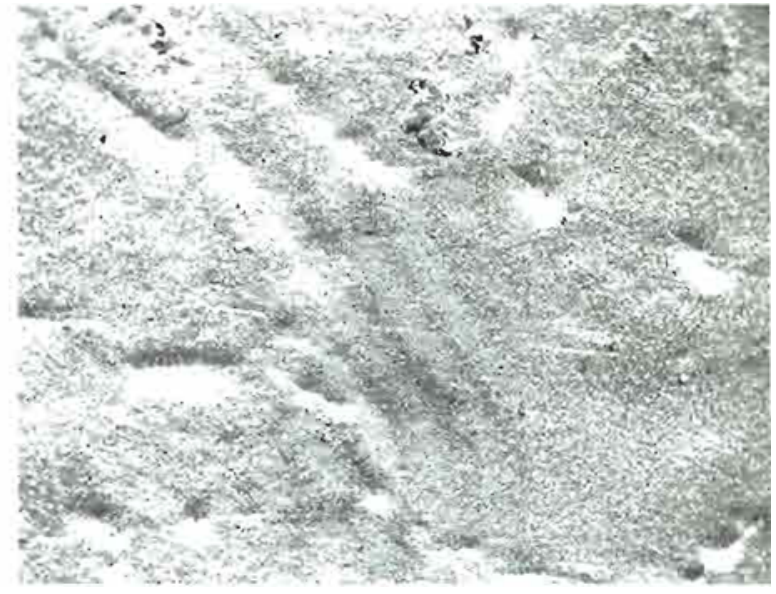

These scanning electron photomicrographs show, clockwise from the top left corner, an immersion gold coating, an electroless gold coating and an clectrodeposited gold conting. All three are viewed under normal incidence. These mierographs do not reveal any signilicant difference between the three types of coatings. Ict, bonding tests performed by the author showed that only the electrodeposited coatings were satisfactory for transistor headers

$\times 650$

backscattering using a platen with a $1.2 \mathrm{~mm}$ opening for the eyelet and using a platen with a $0.3 \mathrm{~mm}$ slot for the through and grounded leads. Scanning electron photomicrographs were taken on the through lead.

In the range of coating thicknesses studied ( 0.26 to $0.76 \mu \mathrm{m}$ ) there proved to be no appreciable cost advantage in the use of electroless as opposed to electrolytic gold deposition. Metal distribution was similar in the two cases.

The solderability of deposits was tested by a standard procedure (2), ageing being replaced by a bake test at $440^{\circ} \mathrm{C}$ in air for two minutes before soldering.

Although the electroless and immersion gold deposits performed satisfactorily in epoxy die and thermocompression or ultrasonic wire bonding, their behaviour in soldering after heating to $440^{\circ} \mathrm{C}$ indicated that diffusion of substrate metal was greater with them than with electrolytically deposited coatings. Voids were also observed in some electroless deposits.

\section{Refierences}

1 Gold Bull., 1979, 12, (3), 106

2 U.S. Military Standard 750 , Method 2026

3 W. S. Rapson and T. Groenewald, Gold Bull., 1975, 8, (4), $119-126$ 